\title{
Coronal Conditions for the Occurrence of Type II Radio Bursts
}

\author{
Athanasios Kouloumvakos ${ }^{1}$, Alexis Rouillard ${ }^{1}$, Alexander Warmuth ${ }^{2}$, Jasmina Magdalenic ${ }^{3,4}$ (10, Immanuel. C. Jebaraj ${ }^{3,4}$, \\ Gottfried Mann ${ }^{2}$, Rami Vainio ${ }^{5}$ (D), and Christian Monstein ${ }^{6}$ \\ ${ }^{1}$ IRAP, Université Toulouse III-Paul Sabatier, CNRS, CNES, Toulouse, France; akouloumvako@ irap.omp.eu \\ ${ }^{2}$ Leibniz-Institut für Astrophysik Potsdam (AIP), An der Sternwarte 16, D-14482 Potsdam, Germany \\ ${ }^{3}$ Solar-Terrestrial Centre of Excellence-SIDC, Royal Observatory of Belgium, B-1180 Brussels, Belgium \\ ${ }^{4}$ Center for mathematical Plasma Astrophysics, Department of Mathematics, KU Leuven, Celestijnenlaan 200B, B-3001 Leuven, Belgium \\ ${ }^{5}$ Department of Physics and Astronomy, University of Turku, FI-20014 Turku, Finland \\ ${ }^{6}$ Istituto Ricerche Solari Locarno (IRSOL), Via Patocchi 57, 6605 Locarno Monti, Switzerland \\ Received 2021 February 1; revised 2021 March 30; accepted 2021 March 30; published 2021 May 28
}

\begin{abstract}
Type II radio bursts are generally observed in association with flare-generated or coronal-mass-ejection-driven shock waves. The exact shock and coronal conditions necessary for the production of type II radio emission are still under debate. Shock waves are important for the acceleration of electrons necessary for the generation of the radio emission. Additionally, the shock geometry and closed field line topology, e.g., quasi-perpendicular shock regions or shocks interacting with streamers, play an important role for the production of the emission. In this study we perform a 3D reconstruction and modeling of a shock wave observed during the 2014 November 5 solar event. We determine the spatial and temporal evolution of the shock properties and examine the conditions responsible for the generation and evolution of type II radio emission. Our results suggest that the formation and evolution of a strong, supercritical, quasi-perpendicular shock wave interacting with a coronal streamer were responsible for producing type II radio emission. We find that the shock wave is subcritical before and supercritical after the start of the type II emission. The shock geometry is mostly quasi-perpendicular throughout the event. Our analysis shows that the radio emission is produced in regions where the supercritical shock develops with an oblique to quasi-perpendicular geometry.
\end{abstract}

Unified Astronomy Thesaurus concepts: Solar radio emission (1522); Solar coronal mass ejections (310); Solar coronal mass ejection shocks (1997)

Supporting material: animation

\section{Introduction}

The occurrence of type II radio bursts has long been related to the acceleration of energetic electrons at shock waves driven by the fast expansion of solar transients such as coronal mass ejections (CMEs; see, e.g., Claßen \& Aurass 2002; Prakash et al. 2009; Magdalenić et al. 2010; Nindos et al. 2011, and references therein). This transient form of radio emission is generated when magnetohydrodynamic (MHD) shocks in the solar atmosphere (Mann \& Classen 1995; Vršnak \& Lulić 2000) accelerate electron beams that excite Langmuir waves owing to beam-plasma instabilities (Knock et al. 2001; Cairns et al. 2003; Schmidt \& Cairns 2012; Mann et al. 2018). The Langmuir waves can be converted by nonlinear wave-plasma processes to radio waves, observable as electromagnetic emission close to the local plasma frequency (fundamental) and/or its harmonic frequencies (Wild et al. 1954), resulting in type II radio emission (Wild \& McCready 1950). Type II radio emission is observed in dynamic spectra (frequency-time diagrams) as slowly drifting emission lanes. It can cover a broad spectral range, from centimetric to hectometric wavelengths, and it lasts from a few minutes to several hours (Nelson \& Melrose 1985). The speed of the exciter, i.e., shock wave, is usually inferred from the type II drift rate and

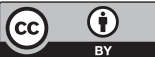

Original content from this work may be used under the terms of the Creative Commons Attribution 4.0 licence. Any further distribution of this work must maintain attribution to the author(s) and the title of the work, journal citation and DOI. assuming a 1D coronal electron density model (i.e., Leblanc et al. 1998; Mann et al. 1999).

Sometimes, it is possible to separate type II radio emission into different components: (1) based on emission bands corresponding to the fundamental and harmonic frequencies, and (2) clearly distinguishable bands of radio emission whose frequency separation is smaller than that of the fundamental and harmonic so-called type II band split. For those radio events that are characterized by a band split (e.g., Vršnak et al. 2001; Zimovets et al. 2012), the separate lanes may have a similar morphology and/or variations in intensity. Different theories have been considered in explaining band split (McLean 1967; Smerd et al. 1975; Holman \& Pesses 1983; Treumann \& Labelle 1992). The most frequently used theory considers band split to result from the nearly simultaneous radio emission from regions upstream and downstream of the shock waves (Smerd et al. 1974, 1975). Assuming that the Smerd et al. (1975) theory for type II band split is correct, the shock density compression ratio can be inferred by the relative separation of the lanes. There are, however, some arguments against the emission from the downstream region of a shock owing to the lack of suitable electron beams to generate Langmuir waves and hence radio emission (Schmidt \& Cairns 2012).

CMEs, flares, and erupting filaments or loops can be considered as the driving agents of the shock waves that are related to type II radio bursts (see, e.g., Warmuth et al. 2004; Nindos et al. 2008, 2011; Magdalenić et al. 2010, 2012; Warmuth 2015; Grechnev et al. 2016, 2018; Kumar et al. 2016; 
Jebaraj et al. 2020). Thanks to the ultra-high-cadence and arcsecond-level EUV imaging of the Atmospheric Imaging Assembly (AIA; Lemen et al. 2012) on board Solar Dynamic Observatory (SDO), together with white-light (WL) and radio imaging (when radio heliographic observations are available), the relationship between type II radio emission and the erupting features can be established with more confidence (Kouloumvakos et al. 2014; Zimovets et al. 2012; Zucca et al. 2014). Recent studies have shown that shock waves are capable of initiating type II radio bursts as long as certain conditions of the ambient coronal plasma are fulfilled (e.g., Zucca et al. 2018; Frassati et al. 2019).

Currently, it is not completely understood which exactly are the conditions necessary for the generation of metric and decametric type II radio bursts and why some fast CMEs, which presumably drive shock waves, are not associated with type II radio bursts, while other slower events do have type II bursts associated with them (e.g., Gopalswamy et al. 2008, 2010; Suresh \& Shanmugaraju 2015). The speed of the exciters and the shock strength should play an important role (Kouloumvakos et al. 2014; Zucca et al. 2018; Krupar et al. 2019); however, it is not a sufficient criterion for the occurrence of type II emission (e.g., Maguire et al. 2020). Recent studies have shown that type II radio bursts could be produced in regions where the shock waves propagate into or close to streamers (see, e.g., Mancuso \& Raymond 2004; Magdalenić et al. 2014; Zucca et al. 2018; Frassati et al. 2019; Jebaraj et al. 2020). In this case the quasi-perpendicular shock geometry could also be a key parameter for efficient particle acceleration (e.g., Cho et al. 2011; Kong et al. 2015), especially for electrons. As discussed by Mann et al. (2018), electron acceleration in a quasi-perpendicular shock wave should be very efficient through the process of shock drift acceleration. These accelerated electrons could be consequently responsible for producing the observed type II radio emission.

In this study we examine a solar event on 2014 November 5 that included a strong flare, a CME, a complex type II radio burst, and, as we shall show, a rapidly evolving shock wave. First, we present an overview of the solar eruptive event and discuss some basic aspects of the observations of the solar event in EUV and radio waves in Section 2. A detailed 3D modeling of the shock wave and estimation of its properties during the expansion in the low corona is presented in Section 3. From the evolution of the shock wave parameters in $3 \mathrm{D}$, we examine the shock properties near the start and end of the type II radio burst (Section 3.3). We then determine the critical parameters that lead to efficient electron acceleration and the production of type II radio emission (Section 4).

\section{Overview of the Solar Eruption}

The event on 2014 November 5 was associated with a GOES M7.9 flare, a filament eruption, and a CME originating from active region NOAA AR 12205 at location N20E68. The flare start, peak, and end times were 09:26, 09:47, and 09:55 UT, respectively, as reported in the online NOAA daily solar event lists and the GOES X-ray light curves from LMSAL. ${ }^{7}$ The flare was also accompanied by a slow CME that was observed by the $\mathrm{SOHO} / \mathrm{LASCO}$ coronagraph with a central position angle (PA) of $87^{\circ}$, a projected line-of-sight speed of $386 \mathrm{~km} \mathrm{~s}^{-1}$, and

7 https://www.lmsal.com/solarsoft/

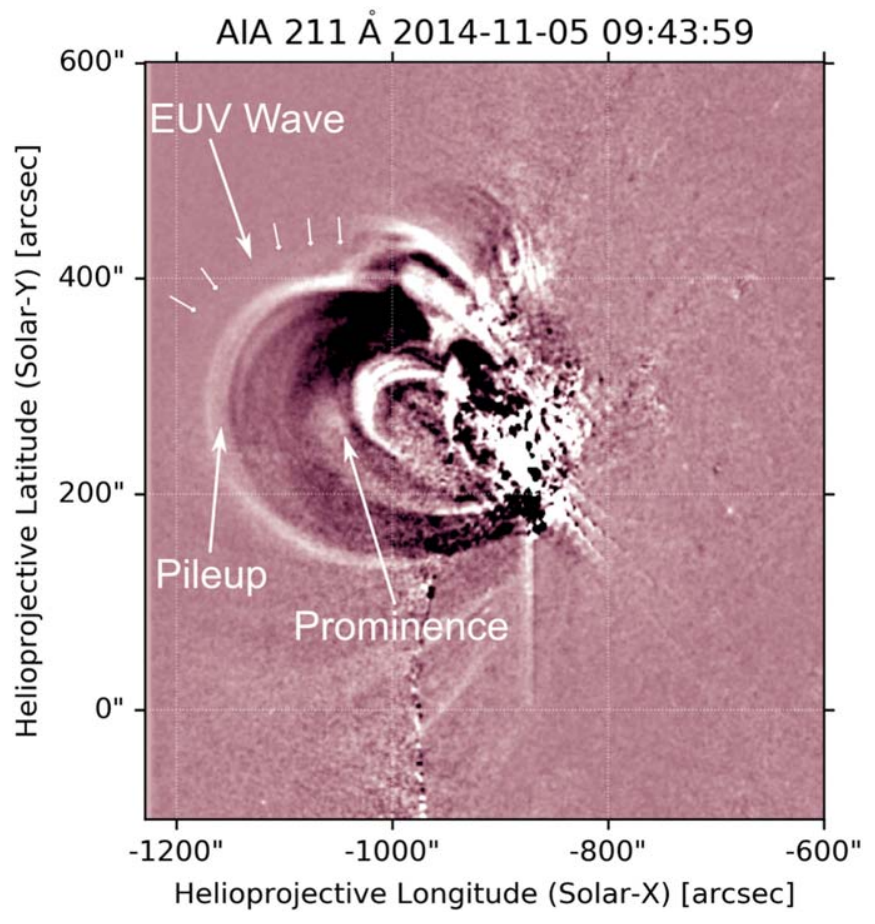

Figure 1. AIA running-difference image at $211 \AA$, during the early stages of the eruption at 09:43:59 UT. The EUV wave, the pileup in front of the CME, and the prominence are indicated.

a width of $187^{\circ}$, as reported in the Coordinated Data Analysis Workshops (CDAW) online catalog. ${ }^{8}$

\subsection{EUV Observations}

The eruption was well observed by AIA/SDO and can be clearly seen from the EUV running-difference images presented in Figure 1. At the early stages of the eruption, a lowlying set of loops lost its equilibrium and started to expand rapidly. The outward-moving material, in the central part of the active region, pushed the initially static overlying magnetic arcade. This phase is best observed in the hot channels of AIA, e.g., at 94 and $131 \AA$, starting at around 09:39 UT. During the eruption, a slowly moving bright core was observed by the AIA 193 and $211 \AA$ channels, which indicates the presence of prominence material. This feature is best observed from 09:44 to 09:47 UT. An associated EUV wave was also observed starting at 09:43:35 UT and was observed better in the AIA $211 \AA$ channel, surrounding the bright erupting system of loops (see Figure 1). The wave expansion can be visually followed in both the radial and lateral direction away from the active region. In the lateral direction, the wave seems to traverse several coronal structures, especially along the northern direction of propagation, where a coronal streamer is located. The interaction of the EUV wave with the streamer and a possible small deflection of the streamer seem to take place at around 09:45 UT (best observed at 193 and $211 \AA$ channels of AIA).

\subsection{Radio Observations}

A composite dynamic radio spectrum of the event is shown in Figure 2. It is constructed by using data from several ground-

\footnotetext{
8 http://cdaw.gsfc.nasa.gov/
} 


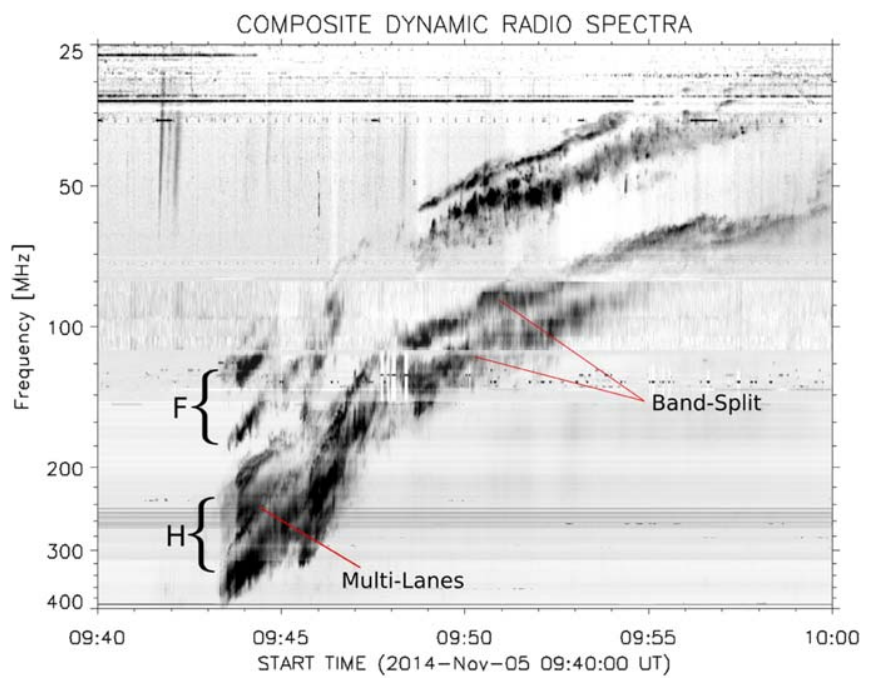

Figure 2. Dynamic radio spectrum in the $25-400 \mathrm{MHz}$ range, composed from NDA, ORFEES, and e-Callisto radio observations during the solar event. The fundamental and harmonic bands of the type II burst are annotated with F and $\mathrm{H}$, respectively.

based radio spectrographs. In the metric wavelengths we used observations from the ORFEES radio spectrograph (which operates at Nançay Radio Observatory) and complemented them with observations from e-Callisto 9 (Birr observatory in Ireland and Bleien radio telescopes in Switzerland). In the decametric wavelengths, we used observational data from the Nançay Decameter Array (Lecacheux 2000). To enhance the visibility of the type II emission, we performed a background and radio-interference subtraction. Additionally, to match the dynamic ranges of the observed emissions from the different radio spectrographs, we rescaled the intensity values to achieve the best visual match of average background intensities for every spectrum.

The onset of type II radio emission is observed at 09:43:30 UT ( $\sim 400 \mathrm{MHz}$ in harmonic emission), and the overall duration is $\sim 17$ minutes (emission ends at around 10:00 UT). No hectometric-kilometric observations were available from the WAVES experiments (Bougeret et al. 1995, 2008) on board Wind and STEREO. Due to this lack of data, we cannot conclude whether there was an extension of the radio emission in the interplanetary range. However, the type II burst does not seem to continue into the hectometric wavelengths, because its intensity strongly decreases when approaching the lowest frequencies of the dynamic spectrum presented in Figure 2. Overall, the type II radio burst consists of multiple lanes, and some of them show the band-split characteristic. Both the fundamental and the second harmonic plasma emission bands (FE and HE hereafter) are well observed. No strong type III bursts were observed during the radio event, apart from a few weak type III bursts observed $\sim 1.5$ minutes prior to the onset of the type II burst.

From the composite radio spectrum of Figure 2 it is clear that type II radio emission in this event is highly complex. Lv et al. (2017) separate the multiple-lane type II radio emission into three episodes (phases). The onset times of those phases are around 09:43:20, 09:45:31, and 09:48:20 UT, respectively (see their Figure 1(b) and their discussion). The characteristic of the band split for some lanes is observed throughout the event, but

\footnotetext{
http://www.e-callisto.org/
}
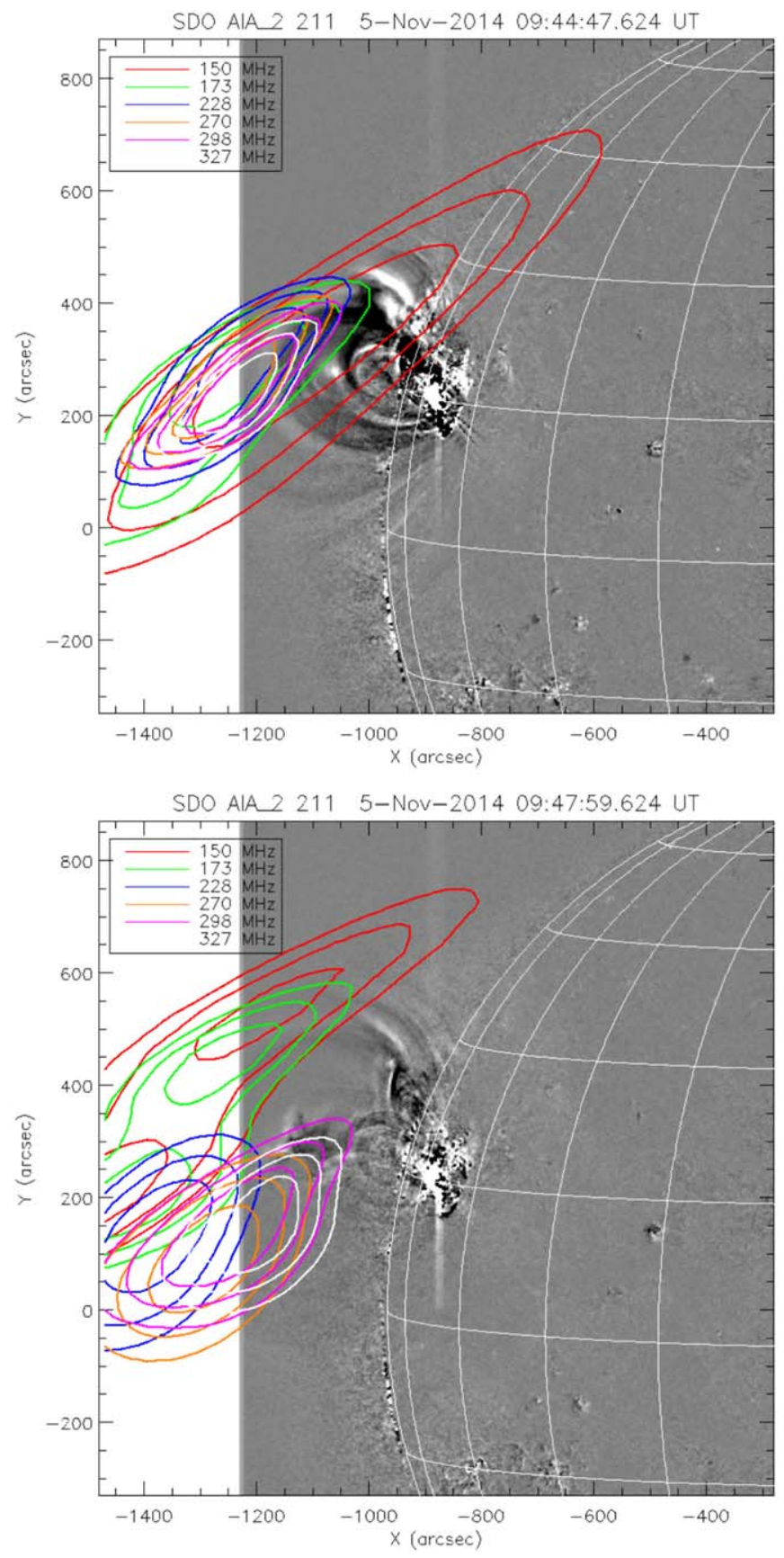

Figure 3. Contours of radio emission from eight different NRH frequencies superposed to running-difference EUV images observed by AIA at $211 \AA$.

it is better distinguishable during the third phase (from 09:48 UT onward). In the early stages of the type II evolution it is difficult to group the different type II lanes into fundamental-harmonic and band-split pairs. After 09:46 UT type II consists of two clearly separable $F-H$ bands, each of which exhibits a clear band split.

Observations from the Nançay Radioheliograph (NRH; see Kerdraon \& Delouis 1997) were also available during the studied event. These observations allow us to examine the radio source locations of the type II burst in the plane of the sky and establish a first connection with the EUV observations. The contours of the type II radio sources observed by NRH are projected on top of AIA EUV images at $193 \AA$ (Figure 3 ) for two selected time intervals. Overall, the evolution of the radio 
source locations seems consistent with the evolution of the pressure wave in the low corona. Namely, in the early phase of the solar event and the type II burst, at around 09:44:42 UT, the source of the type II emission is primarily located close to the apex of the expanding wave and slightly shifted toward the southeastern flank. This can be seen in the top panel of Figure 3.

After 09:45 UT, the radio emission progressively becomes more extended around the wave, with larger sources and simultaneous multiple source locations. From the bottom panel of Figure 3 it is apparent that the emission is mostly localized near the wave apex, but there are also sources of radio emission near the flanks of the wave. One is located at the western flank, and another is located near the northeastern flank. The multiple source locations of the type II emission at this stage seem consistent with the complex multiple-lane type II emission observed at the same time (see the composite radio spectra of Figure 2). We will return to these aspects in Section 4, where we will examine in more detail the source location of the radio emission in connection to the 3D shock model.

\section{Shock Wave Modeling}

\subsection{The Shock Wave Parameters in $3 D$}

We reconstruct the shock wave and compute its parameters by using the methods presented in Rouillard et al. (2016) and Kouloumvakos et al. (2019). Those techniques start from the $3 \mathrm{D}$ reconstruction of the observed pressure wave. The wave reconstruction in the low corona is usually performed by using full-disk EUV images at $195 \AA$ from the Extreme UltraViolet Imager (EUVI; Wuelser et al. 2004) on board STEREO and $193 \AA$ images from SDO/AIA. Higher in the corona, the reconstruction is done using total brightness coronagraph image triplets from the Sun Earth Connection Coronal and Heliospheric Investigation (SECCHI; Howard et al. 2008) coronagraphs on board STEREO and the Large Angle and Spectrometric Coronagraph (LASCO; Brueckner et al. 1995) coronagraphs on board SOHO. Unfortunately, no observations were available from the STEREO spacecraft at the time of the event. Therefore, the reconstruction is performed using only single-viewpoint observations from SDO and $\mathrm{LASCO} / \mathrm{C} 2$. The wave degenerates well below the inner field of view of $\mathrm{LASCO} / \mathrm{C} 3$, and so we stopped the modeling at LASCO/C2 heights. Nevertheless, the type II radio burst ends well before the inner field of view of LASCO/C3.

To model the parameters of the pressure wave in $3 \mathrm{D}$, we calculate, as a first step, the wave expansion speed in 3D from the reconstructions of the observed pressure wave. Next, we use MHD data from the Magneto-Hydrodynamic Around a Sphere Thermodynamic (MAST) model (provided by Predictive Sciences Inc.; see Lionello et al. 2009; Riley et al. 2011) to infer the upstream plasma and magnetic field properties along the surface of the pressure wave. The MAST model is a 3D MHD model that employs photospheric magnetograms from SDO/HMI as the inner boundary condition of the magnetic field and includes detailed thermodynamics with realistic energy equations accounting for the thermal conduction parallel to the magnetic field, radiative losses, and parameterized coronal heating. Previous studies have shown that the MAST model is capable of reproducing well the global coronal features observed in WL, EUV, and X-ray emission (Lionello et al. 2009; Rušin et al. 2010).
We also calibrate the 3D magnetic field and density data obtained from the MAST model, in order to achieve a better match with the observations. For the magnetic field we use the method presented in Rouillard et al. (2016; see also Kouloumvakos et al. 2019). By comparing the radial magnetic field components of the MHD cubes at the outer boundary with the values measured near $1 \mathrm{au}$, we find a correction factor of $\approx 1.5$ for the magnetic field. For the calibration of the density data, we performed a differential emission measure analysis of the SDO/AIA images and compared the results with the MHD data. For this we used the method of Aschwanden et al. (2013), which is implemented in SolarSoft ${ }^{10}$ in the aiateemmap2.pro routine. Aschwanden et al. (2013) perform a forward-fitting approach to solve the EM problem assuming a Gaussian or combinations of Gaussians for the emission measure as a function of temperature $(\log (T))$ and then vary the parameters of the assumed emission measure until the resulting intensity best matches the observed AIA intensity. See Aschwanden et al. (2013) and Aschwanden \& Boerner (2011) for further details. The electron density can then be obtained from the emission measure of the maps (e.g., Zucca et al. 2014). We have used a constant line-of-sight path length, since it does not change significantly in the lower coronal range $\left(1-1.3 R_{\odot}\right.$; see Zucca et al. 2014, for further details). From this analysis we find that a correction factor of $\approx 2.2$ has to be applied to densities computed by the MHD model at the heights and regions we focus on this study.

Using the 3D wave expansion speed and the upstream MHD parameters from MAST, we calculate the Mach number (Alfvén mach number $M_{\mathrm{A}}$ and fast magnetosonic Mach number $\left.M_{\mathrm{fm}}\right)$, the density compression ratio $(X)$, and the magnetic field obliquity with respect to the shock normal $\left(\theta_{\mathrm{BN}}\right)$ (see Kouloumvakos et al. 2019). We note that, because of the correction applied to the density and the inverse dependence of the Alfvén speed on the plasma density, the computed Mach number for some shock regions could be a higher estimate. Using the vector magnetic field data obtained from the MAST $3 \mathrm{D}$ cube, we also perform field line tracing. This allows us to examine the topology of the magnetic field through which the shock wave expands.

In Figure 4 we show a 3D view of the reconstructed pressure wave at 09:46:00 UT and the magnetic field lines traced from the MAST model. The color maps plotted on the surface of the wave show the calculated $M_{\mathrm{A}}$ (panels (a) and (b)) and the $\theta_{\mathrm{BN}}$ (panels (c) and (d)) values. Using the information obtained from the shock wave modeling and the magnetic field line tracing, we show that the shock wave evolves near a coronal streamer. This is where the open (blue) and the closed (red) field lines meet at the streamer boundaries in Figure 4. The shock strength, as quantified by $M_{\mathrm{A}}$, is relatively high at the apex and over a broad region extending from north to south as labeled in Figure 4. The highest shock strength is observed at the boundary between open and closed field lines (Figure 4 (b)). This is near the apex and adjacent to it in the northward direction with $M_{\mathrm{A}} \sim 3.9$.

As the CME/flare event takes place in a region with primarily closed magnetic field topology (see Figures 4(e) and (f)), the shock geometry is mostly oblique to quasi-perpendicular. The highest value of $\theta_{\mathrm{BN}}$ is found over a broad region starting from the shock apex and toward the core of the

\footnotetext{
$\overline{10}$ https://sohowww.nascom.nasa.gov/solarsoft/
} 

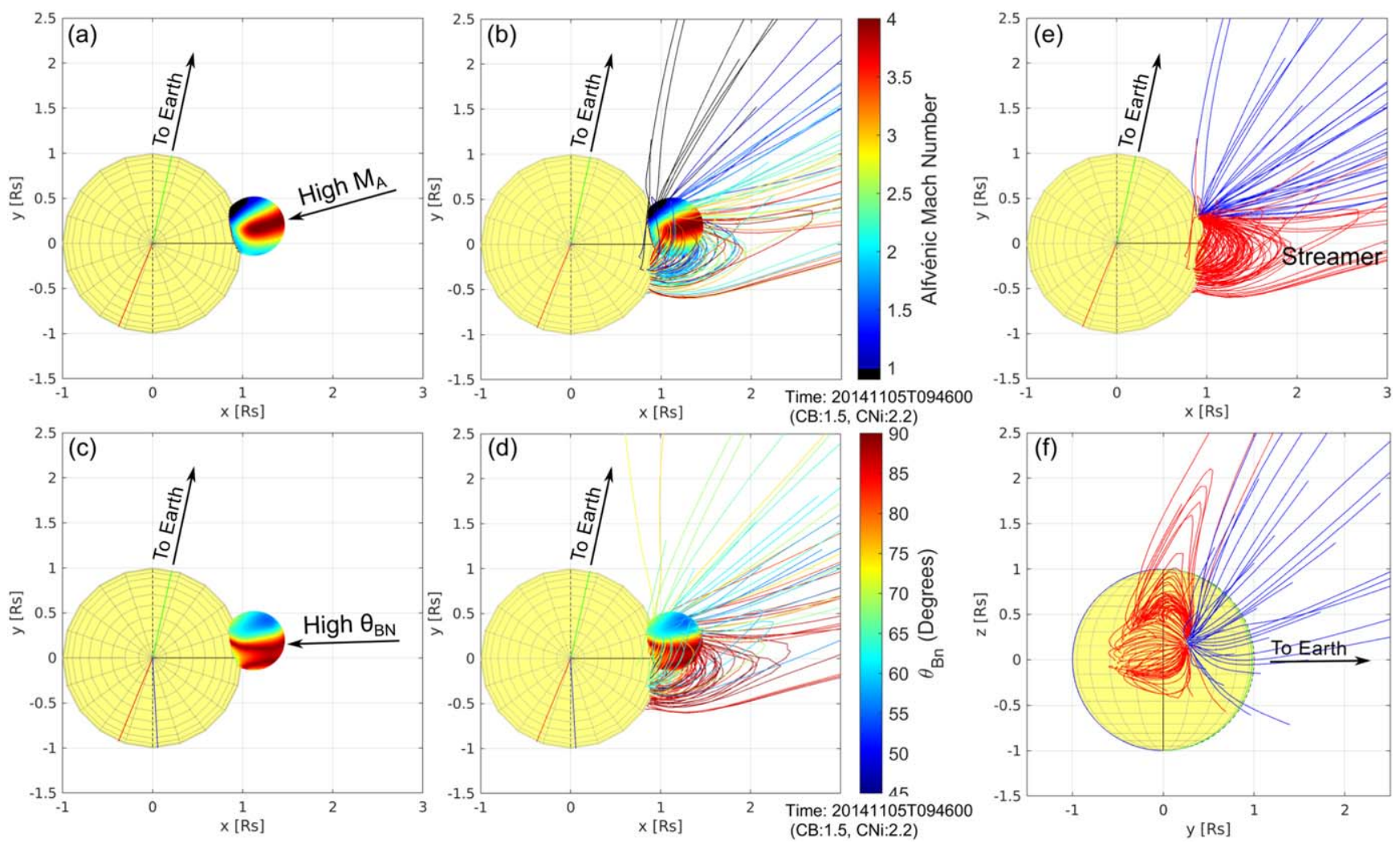

Figure 4. Results of the modeled shock wave parameters in 3D plotted along the reconstructed pressure wave front surface around 09:46:00 UT. Panels (a) and (b) show the distributions of the modeled $M_{\mathrm{A}}$ and panels (c) and (d) the distributions of the modeled $\theta_{\mathrm{BN}}$ along the front surface, with and without the field lines traced from the MAST model. For display purposes only a fraction of the total field lines that are connected to the wave surface are plotted in panels (b) and (d). The coloring of the field lines is analogous to the shock parameter value at the point where the field line threads the shock surface. Panels (e) and (f) show 3D views from different viewing directions of the traced field lines. Open and closed field lines are depicted in blue and red. The central solar meridian, as viewed from Earth and STEREO-A, is shown with green and red arcs, respectively. (An animation of panels (b) and (d), running from 09:44:30 to 09:49:30 UT, is available in the online Journal.)

(An animation of this figure is available.)

streamer. In this region, the shock is quasi-perpendicular, with $\theta_{\mathrm{BN}}$ values greater than $75^{\circ}$. For the regions outside of the streamer, the shock is mostly oblique, with $\theta_{\mathrm{BN}}$ ranging between $45^{\circ}$ and $65^{\circ}$. As we discussed in Section 1, the quasiperpendicular geometry favors an efficient acceleration of electrons through the shock drift acceleration mechanism. It is also worth noting that the 3D characteristics, such as shock strength and shock geometry, show significant evolution during the time line of the event.

The shock wave initially expands in a region with primarily closed magnetic field topology and later propagates in a region with both open and closed magnetic field lines near the streamer boundaries. This can be better visualized in the animation provided with Figure 4 (see also the relevant discussion for Figure 5 in the next section).

\subsection{Evolution of the Shock Wave Parameters}

We start this analysis by examining the evolution of the shock parameters throughout the event. For each of the resultant shock parameters from the shock modeling, we calculate the time history of the distribution characteristics (mean, median, and first/third quartile and decile values) for the regions in which a shock wave has probably formed $(X>1)$. The time history of the $M_{\mathrm{A}}, M_{\mathrm{fm}}$, compression ratio, and $\theta_{\mathrm{BN}}$ is shown in Figure 5, from 09:42 to 10:04 UT. Parts of the wave were already shocked before the onset of the type II radio burst, which was around 09:43:30 UT (see Figure 2).
Those regions are located mainly at the apex where the Mach number and compression ratio are greater than unity; however, the shock is not supercritical (e.g., $M_{\mathrm{A}} \leqslant 2.1$; see also the discussion in Section 3.3). This result is also in accordance with the previously discussed observational findings indicating that only a fraction of fast CME-driven shocks is associated with the type II emission. The existence of the quasiperpendicular shock is a necessary condition for the type II formation, but not a sufficient one.

From the beginning of our shock modeling at 09:42 UT and for several minutes onward, the shock strength increases until around 09:46 UT, when a maximum is reached (see Figure 5(a) and (b)). At this time, the median $M_{\mathrm{A}}$ is $\sim 2.31$ and $X \sim 2.25$, and we find that the shock regions located near the shock's apex are supercritical and are having the maximum strength. Toward the shock flanks, at the northern/southern parts, the shock strength is lower; however, as we show in Figure 4, those regions are also supercritical. On the eastern/southern flanks, the shock is mainly subcritical. ${ }^{11}$ After the maximum at $\sim 09: 46 \mathrm{UT}$, the shock strength quantified by $M_{\mathrm{A}}$ and compression ratio remains almost constant, while the median $M_{\mathrm{fm}}$ decreases slightly from around 2.03 to 1.65 (see Figure 5(c)) and remains around this value until the end of the shock modeling.

\footnotetext{
${ }^{11}$ For an oblique to quasi-perpendicular shock geometry, which is mostly the case of the modeled wave in this study, the critical Mach number for a shock wave to be supercritical is around 2 .
} 

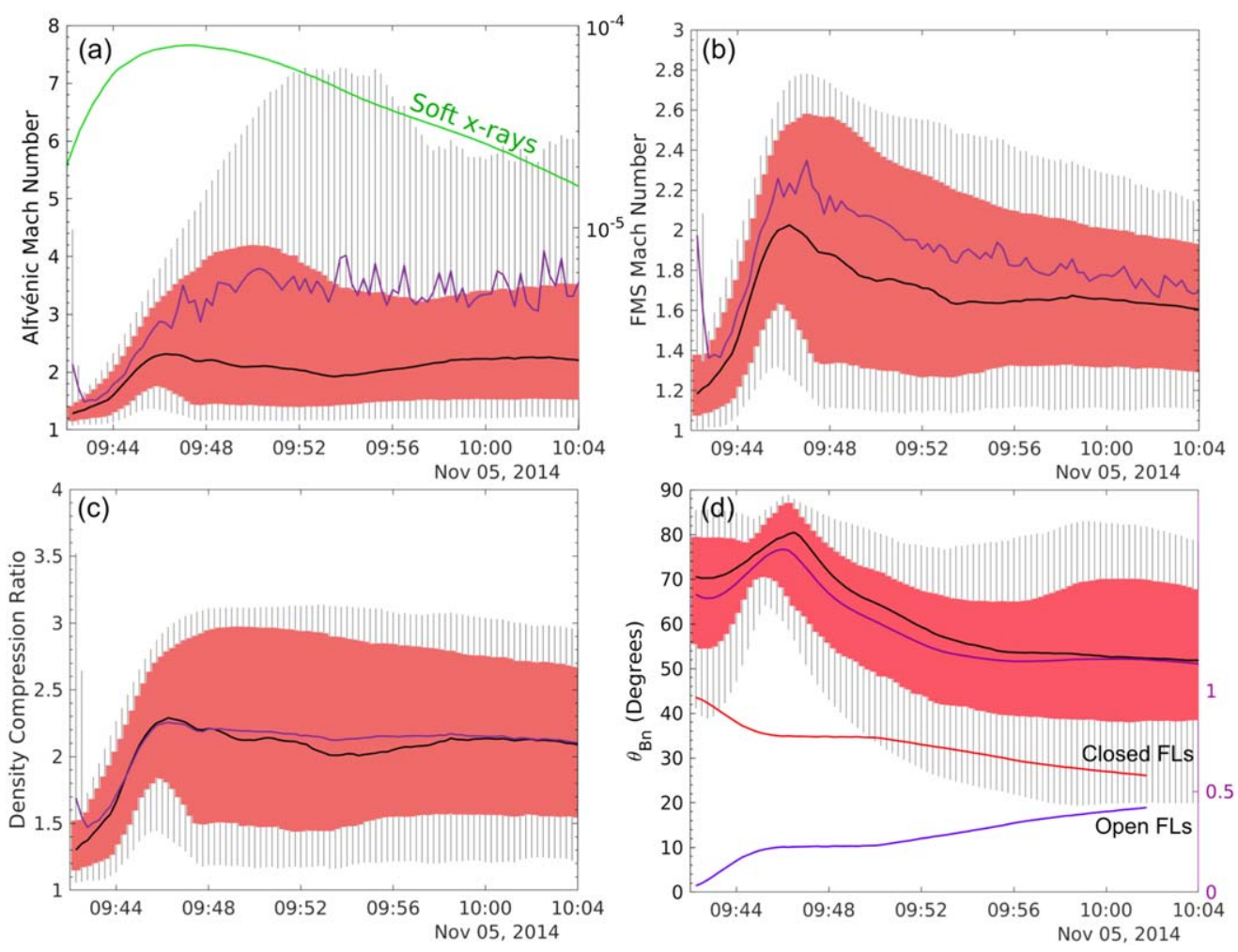

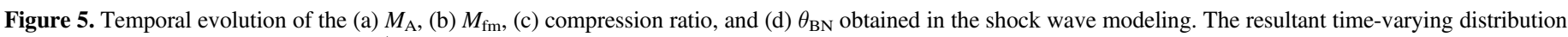

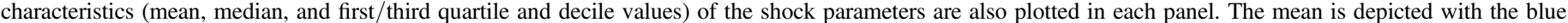

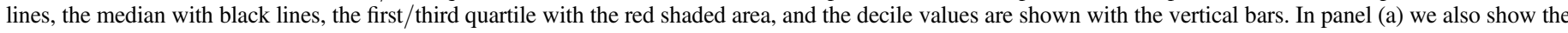

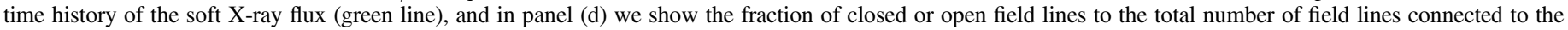
shock surface.

Further, we examine the temporal evolution of the magnetic field obliquity with respect to the shock normal $\left(\theta_{\mathrm{BN}}\right)$. This parameter provides additional information about the shock wave geometry near the onset of type II and throughout the event. The temporal evolution of the $\theta_{\mathrm{BN}}$ is presented in Figure 5(d). The shock geometry at the beginning of the shock modeling is quasi-perpendicular, with $\theta_{\mathrm{BN}}$ values around $70^{\circ}$. Similar to the shock strength, the shock geometry rapidly changes in several minutes. The value of $\theta_{\mathrm{BN}}$ increases until 09:46:30 UT, when the shock wave geometry is closest to being perpendicular with a median value of $\sim 80^{\circ}$. The shock wave geometry then evolves toward a more oblique state with values of $\theta_{\mathrm{BN}}$ close to $50^{\circ}$, where this value remains until the end of the shock modeling. As we have shown in the previous section, this rapid variability of the shock geometry throughout the event is related to the shock wave evolving inside the streamer region.

Figure 5(d) also includes the time history of the fraction of closed and open field lines to the total number of field lines that thread the surface of the shock wave. The shock wave expands partially inside the streamer loops during the early stages of its evolution, where the magnetic field topology in front of the shock wave is dominated by closed field lines (see, e.g., Figure 4). At the beginning of the shock modeling and for a few minutes later, we find that $\sim 90 \%$ of the field lines connected to the shock wave are closed field lines as shown in Figure 5(d). The fraction of closed field lines connected to the shock constantly decreased throughout the event as the shock wave expanded away from the Sun. Near the end of the event, at around 10:04 UT, the fraction of closed and open field lines connecting to the shock surface was equal.

\subsection{Shock Wave Properties near the Onset and End of Type II}

We also examine the shock wave properties in 3D space near the onset and at the end of the type II radio burst (Figure 6). The different shock parameters are represented with color maps plotted on the surface of the shock wave before (panels (a)-(c)) and after (panels (d)-(f)) the onset, and finally at the end (panels (g)-(i)) of the type II radio burst. The viewpoint has been adjusted in such a way that most of the relevant and discussed features of the shock wave are visible. The central solar meridian (as seen from Earth) is denoted by the green arc.

The 3D distribution of the compression ratio near the onset of the type II radio burst is presented in Figures 6(b) and (e). As noted in Section 3.2, we find that a shock wave formed before the onset of the type II radio burst mainly along the apex and parts of the flank of the shock ellipsoid. For the stronger shock regions located at the apex, we found that the density compression ratio changes from $\sim 2$ before the onset of the type II burst to $\sim 2.6$ after the onset. For the regions located below the wave flanks that are shaded in black, the density compression ratio is less than unity. It is unlikely that a shock wave was formed in these regions during the time interval discussed here.

The 3D distribution of the $M_{\mathrm{A}}$ at the same time interval (see Figures 6(a) and (d)) indicates that the strongest shock regions are located near the apex, and the evolution of $M_{\mathrm{A}}$ at those regions shows that the shock becomes supercritical very close to the start time of type II radio emission. At around 

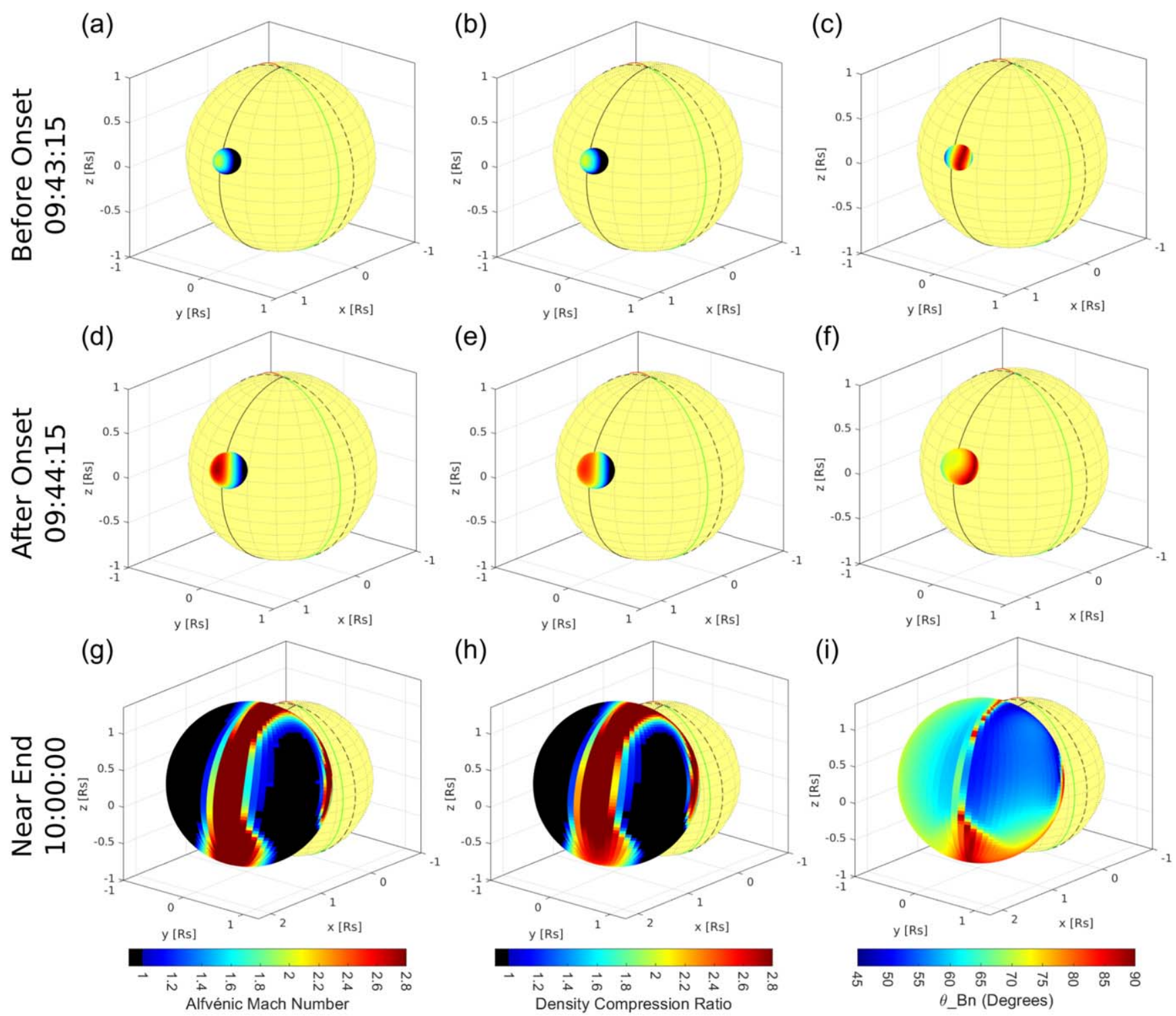

Figure 6. Results of the modeled shock parameters in 3D, overplotted along the reconstructed pressure wave front surface for three selected time intervals. Top panels show the modeled shock parameters before type II onset, middle panels show after type II onset, and the bottom panels show near the apparent end of the type II radio burst. The distributions of the modeled $M_{\mathrm{A}}, X$, and $\theta_{\mathrm{BN}}$ are shown in the left, middle, and right panels, respectively, for each time interval.

09:43:15 UT, e.g., just before type II onset, we found that the highest $M_{\mathrm{A}}$ near the apex was $\sim 1.6$, while after type II onset at 09:44 UT, the shock strength increases to $\sim 2.5$. The shock geometry in the same regions was on average quasi-perpendicular, $\theta_{\mathrm{BN}}>65^{\circ}$. The changes of the shock geometry that we described in Section 3.1 are also evident in Figures 6(c) and (f).

Near the end of the type II radio burst, the shock wave is strong along an extended region that forms a meridional band extending all around the shock. This region of the shock wave is mainly located inside the streamer, where the Alfvén speed is low, the plasma density is high, and the magnetic field is weak. As a consequence, the shock strength and the compression ratio are enhanced. The shock geometry has also changed significantly compared to the start of the event. The value of $\theta_{\mathrm{BN}}$ is less than $45^{\circ}$ in most parts of the shock wave, and so the shock wave geometry is mainly oblique to quasi-parallel, and only in the region close to the southern flank is the shock quasiperpendicular.
Hence, we can conclude that the start of type II radio emission is consistent with the stage when the shock wave becomes supercritical. We also found that the shock geometry mostly remains quasi-perpendicular during this time. The transition to a predominantly quasi-parallel shock geometry may explain the apparent end of the type II radio burst at around 10:00 UT. We also compare regions where the shock wave is strong and quasi-perpendicular with the locations where the type II radio sources (Figure 3) were found during the start of the type II radio burst. The NRH observations indicate that the source regions of type II during its onset are near the apex and toward the southern flank. We discuss this further in Section 4.

\section{Connecting the Shock Modeling with the Evolution of Type II Emission}

In the analysis presented in Section 3.2, we showed that the evolution of the shock wave parameters (strength and geometry) seems to be broadly consistent from the start until 

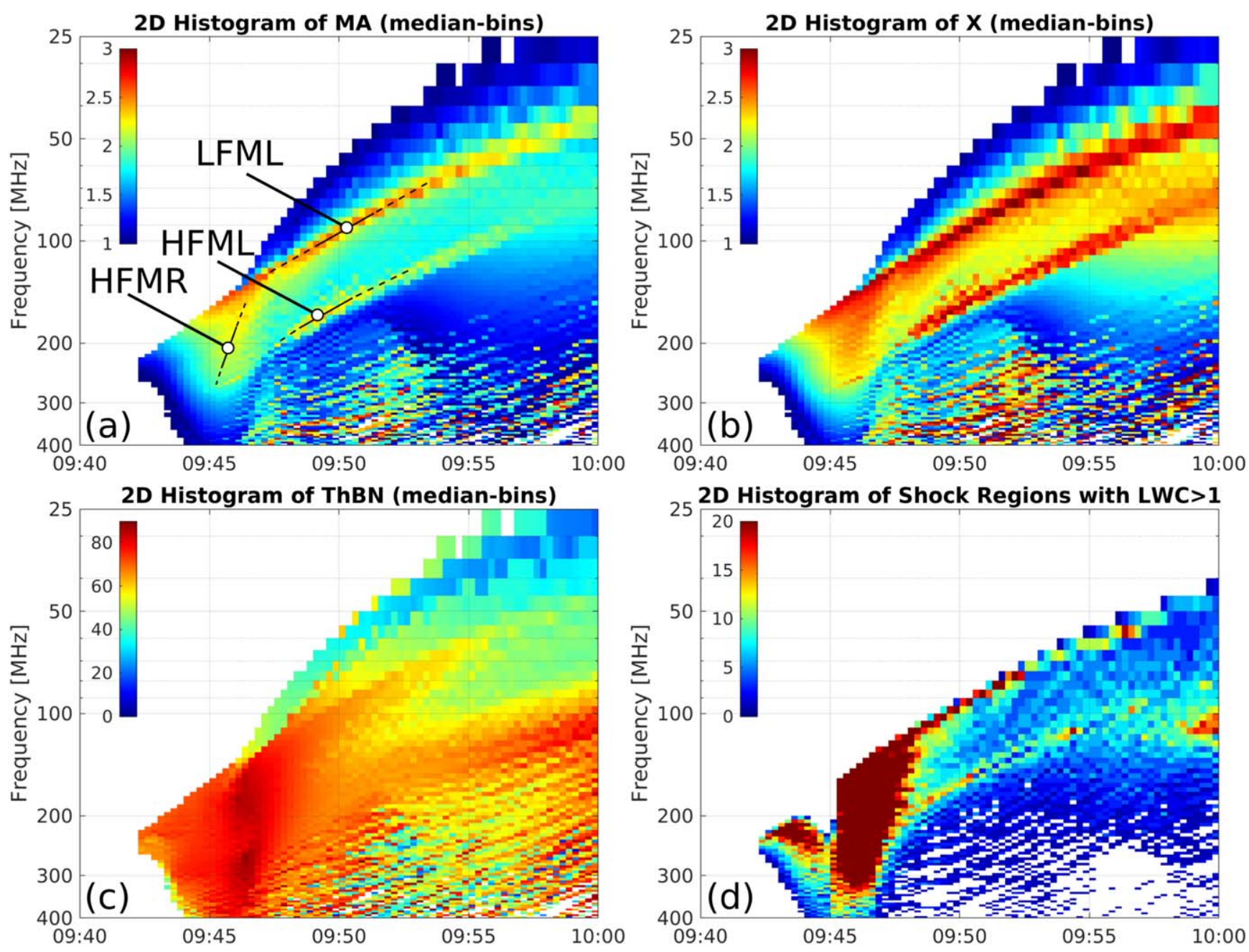

Figure 7. 2D-binned histogram maps of different shock parameters using a dynamic spectrum-like representation (more details for the construction of the maps are given in the text). Panel (a) shows the synthetic spectra for $M_{\mathrm{A}}$, panel (b) for the density compression ratio, panel (c) for $\theta_{\mathrm{BN}}$, and panel (d) for the regions where the criterion for the excitation of Langmuir waves is fulfilled. The intensity of the synthetic spectra depicts the median values of the shock parameter considered for each panel. The high-intensity features labeled in panel (a) as low-frequency modeled lane (LFML), high-frequency modeled lane (HFML), and high-frequency modeled region (HFMR) are regions of high median shock strength (see text for details)

the end of type II radio emission. However, it is difficult to discern the shock regions producing the radio emission and to derive specific details about their properties (i.e., strength and geometry) from the 3D shock modeling alone. In this section we introduce a novel method to examine in more detail which shock regions are probably responsible for type II radio emission and what are their characteristics.

Starting from the 3D shock modeling, we use the upstream coronal density and map the different parameters (e.g., $\theta_{\mathrm{BN}}$ and $M_{\mathrm{A}}$ ) into a representation similar to a dynamic spectrogram. The methodology for the production of the maps is detailed as follows: for each time step of the modeled pressure wave we consider the points along the ellipsoid surface that a shock wave has formed $(X>1)$. For those points, we convert the density to frequency (fundamental or harmonic) since $f_{p} \propto\left(n_{e}\right)^{1 / 2}$. In such a way we produce 2D-binned histograms (synthetic spectra from hereon) of frequency versus time. For the intensity of the synthetic spectra we consider the median values of a shock parameter calculated for each bin (e.g., Figure 7)

Figure 7 presents the synthetic spectra of the shock strength (panel (a)), density compression ratio (panel (b)), and shock geometry (panel (c)). The intensity of the maps depicts the median values of the shock parameters calculated only for the shock regions registered at each bin. The mapping of the shock surface points has been done for the second harmonic plasma frequency. The synthetic spectra in Figure 7 show the spectral regions where type II radio emission can be produced from the evolving shock front. The intensity of the synthetic spectra provides us information on the importance of the different shock parameters. For this analysis we have assumed that only shocked regions $(\mathrm{X}>1)$ are capable of producing type II radio emission and that the emission is produced close to the shock front in the upstream region (Cairns et al. 2003).

In all synthetic spectra presented in Figure 7 we observe two lanes of high intensity, i.e., strong shock regions (high $M_{\mathrm{A}}$ and $X$ ) with high $\theta_{\mathrm{BN}}$ values accompanied by a clear frequency drift. These modeled high-intensity lanes (labeled as lowfrequency modeled lane (LFML) and high-frequency modeled lane (HFML) in Figure 7) can be interpreted as follows: as the shock wave evolves inside the streamer region (see, e.g., Figure 4), the shock strength increases locally in the regions where the magnetic field is low and the density is high. The high-intensity drifting region, visible at low frequencies (see 
the labeled LFML in Figure 7), appears to coincide with the low-frequency lane of the type II harmonic band (Figure 2). The frequency range and the drift rate between the observed and the modeled type II are qualitatively similar.

A qualitative comparison of the synthetic spectra presented in Figure 7 with the dynamic radio spectrum (Figure 2), indicates that type II radio emission is produced where the shock wave is strong and supercritical with high-density compression ratio and high $\theta_{\mathrm{BN}}$ values (i.e., quasi-perpendicular regions). Furthermore, the analysis of the radio event (Figure 2) presented in Section 2.2 indicates the presence of a separate type II phase starting around 09:46 UT (see also Lv et al. 2017). The spectral range at which this high-frequency emission is observed in Figure 2 is also the region where the shock strength is very high according to the synthetic spectra (Figure 7). There are strong shock regions mapped at the same high-frequency range as in the observed spectrum (starting at $\sim 300 \mathrm{MHz}$ around 09:46 UT). We label this distinct drifting emission lane the high-frequency modeled region (HFMR) in Figure 7 . The $\theta_{\mathrm{BN}}$ values are also very high $\left(>80^{\circ}\right)$ in HFMR. Figure 5 shows that the median shock obliquity is quasiperpendicular and maximum around 09:46 UT. The shock strength $\left(M_{\mathrm{A}}\right)$ is moderate at this region, with values ranging from 1.7 to 2.3 .

Next, we search for supercritical shock regions that are capable of generating electron beams that could excite Langmuir waves. For the excitation of Langmuir waves the velocity of the electron beam, $v_{b}$, must exceed at least $\sqrt{3}$ times the velocity of thermal electrons, $v_{e}$. The velocity of the electron beam can be determined from $v_{b}=v_{s} \sec \theta_{\mathrm{BN}}\left(1+\cos ^{2} \alpha_{l c}\right)$ (see, e.g., Mann et al. 2018), where $v_{s}$ is the shock speed and $\alpha_{l c}=\arcsin \sqrt{B_{1} / B_{2}}$ is the loss-cone angle, which depends on the ratio between up- and downstream magnetic field strength. In Figure 7(d) we show the 2D-binned histogram for the shock regions for which the criterion for the excitation of Langmuir waves is fulfilled (e.g., $v_{b}>\sqrt{3} v_{e}$ ). All the parameters for this calculation are obtained in the shock model, and we have also assumed the temperature of the upstream electrons $\left(T_{e}\right)$ and protons $\left(T_{e}\right)$ to be approximately the same $\left(T_{e} \approx T_{p}\right)$. The highest contribution is observed from $\sim 09: 45$ to $09: 47$, when both the shock strength and $\theta_{\mathrm{BN}}$ values are nearly maximum.

The synthetic spectra presented in Figure 7 provide important information about the properties of the shock in regions where type II is likely produced. We begin by evaluating the possible differences between the model and the observations. A first inconsistency could be due to the limitations of the shock/MHD models themselves, as they may not capture the full complexity of the shock front and their effects on the production of type II radio bursts as is visible when qualitatively comparing Figure 7 with Figure 2. As shown earlier, the low-frequency lane of the harmonic type II emission band is related to the LFML of the synthetic spectra. However, this is not the case when considering the HFML of the synthetic spectra (Figure 7) when no emission is observed.

There are a few possible explanations for this difference between the modeling and the observations. A first scenario is that the wave regions associated with the HFML are mostly subcritical $\left(M_{\mathrm{A}}<2\right)$, so the final emission from those regions may not be significant. From Figures 7(a) and (b) we see that the LFML is mapped with moderate to high intensities while the HFML is mapped with lower intensities, e.g., the strength is considerably lower and close to the critical Mach number. Further, the HFML lane has a significantly lower bandwidth than the LFML. Additionally, Figure 7(d) shows only a small fraction of the HFML, and this suggests that only a few regions could excite Langmuir waves and produce radio emission. There are some other scenarios that could also explain the inconsistency between the model and the observations for the HFML. Those are discussed in detail in Section 5.

We continue our analysis with a comparison between the shock model and the radio observations from NRH for three selected times. Figure 8 shows the radio source contours from NRH overlaid on top of the modeled shock wave. The top panels show the values of the modeled $M_{\mathrm{A}}$ along the shock surface, and the bottom panels show the modeled $\theta_{\mathrm{BN}}$ values.

Figures 8(a) and (b) present the comparison between radio observation and shock wave modeled at 09:44:45 UT, i.e., 1 minute after the onset of the type II burst. The source region contours of the radio emission are found mostly near the apex of the shock wave, which correlates well with regions where the shock is strong (and supercritical) and its geometry is quasiperpendicular.

A similarly good correlation is found between the shock model and radio observation for the other two time intervals presented in Figure 8. Figures 8(c) and (d) (also, panels (e) and (f)) show the shock wave farther out and the supercritical regimes along its flanks. The NRH radio sources at this time were observed on multiple locations on the shock surface; however, we note that they are consistently found in regions where the value of $M_{\mathrm{A}}$ is high (e.g., strong shock regions) and the shock geometry is oblique to quasi-perpendicular.

\section{Summary and Discussion}

In this study, we performed an in-depth analysis of a pressure wave observed during the 2014 November $5 \mathrm{CME} /$ flare event. We used single-viewpoint EUV and WL observations from SDO and LASCO to reconstruct and model the pressure wave in $3 \mathrm{D}$. The joint analysis of the shock wave parameters was done using the 3D modeling and radio observations to examine the shock wave conditions necessary for the production of type II radio emission. We introduce a novel methodology that enables us to compare and correlate the results of the shock wave modeling with the radio observation of the type II radio burst.

The reconstruction of this event was a complex task since the available observations in EUV and WL were only from a single viewpoint. Nevertheless, the high-cadence EUV imaging from SDO and the moderate wave speed $\left(\sim 700 \mathrm{~km} \mathrm{~s}^{-1}\right)$ worked in favor of a reliable final reconstruction of the wave in both the radial and lateral direction. The only ambiguity in the position of the wave is in the extension of the eastern flank of the wave, which is located behind the eastern solar limb. We have assumed a self-similar expansion in this direction. We next used the reconstructed wave expansion speed in 3D and upstream MHD parameters from the MAST model to calculate in 3D the shock Mach number, the density compression ratio, and the magnetic field obliquity with respect to the shock normal.

The primary focus of the analysis was the spatial distribution and evolution of the shock parameters. Our results show that the shock wave evolves near a coronal streamer, so the coronal regions where the shock propagates are mainly regions with closed field lines (see Figure 5). From the beginning of the 

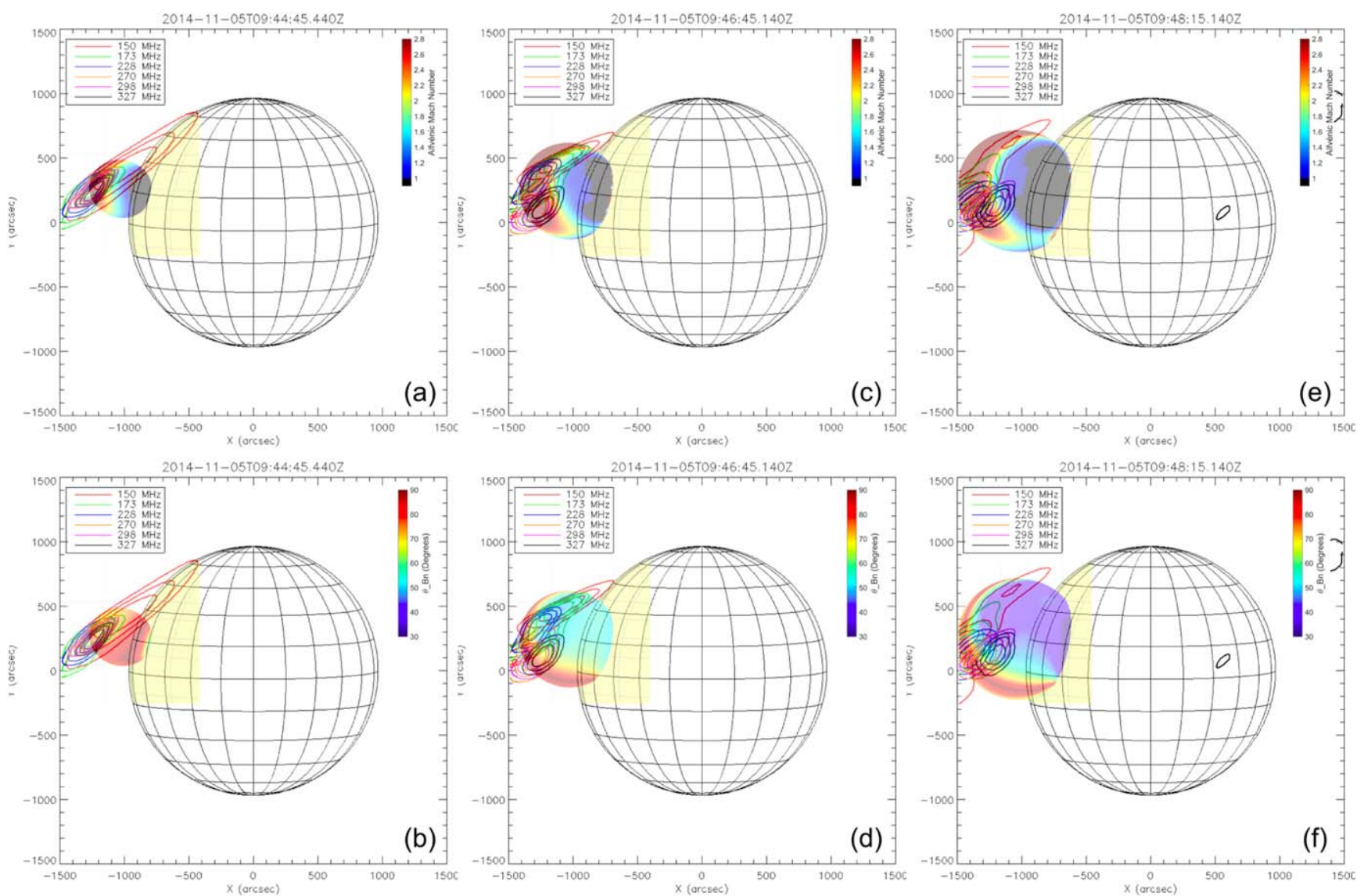

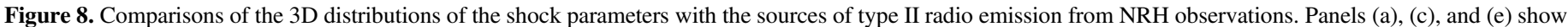

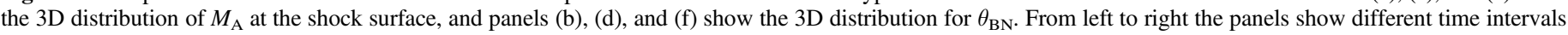
considered for this comparison.

shock model we find regions where a subcritical shock wave has formed; the shock wave becomes on average supercritical some minutes later. The highest shock strength and density compression are found near the apex at around 09:46 UT. The shock geometry is mostly quasi-perpendicular and changing toward oblique values near the end of the modeling. Since the shock wave propagates into a streamer region, there is a rapid spatial and temporal variation of $\theta_{\mathrm{BN}}$ throughout the event.

A shock wave has possibly formed before the start of the type II emission; however, as most of the shock wave regions were subcritical, the associated radio emission was not generated. Near the stage at which the shock wave starts to progressively become supercritical (mainly near the apex), the onset of type II emission is observed. Additionally, at this stage the shock geometry is mainly quasi-perpendicular, and the shock propagates mostly through closed field lines. Therefore, our results suggest that the type II emission is generated when the shock wave becomes supercritical, and thus efficient electron beam acceleration is expected to occur. We find it more difficult to explain the apparent end of the type II radio burst when only considering the evolution of the shock strength since many shock regions continue to be supercritical near the apparent end of the type II emission. We find that the shock wave near the end of type II is oblique. Therefore, it seems that the changes in the shock geometry near the end of the radio event play an important role in the end of the radio emission from the shock regions. Similar conditions for the generation of type II emission have been found in resent studies (Zucca et al. 2018; Maguire et al. 2020).

To investigate further the connection of the shock model with the evolution of the type II emission, we introduce a new methodology that maps the shock parameters into a spectrumlike representation. From the derived synthetic spectra of the different shock parameters we find that type II radio emission seems to be produced at regions where the shock wave is strong and supercritical, with a high-density compression ratio and also high $\theta_{\mathrm{BN}}$ values (i.e., quasi-perpendicular geometry). All those conditions are consistent with the scenario in which type II radio emission, for the event studied here, is produced at the strongly shocked region that propagates inside the streamer. The coronal conditions seem to play an important role for the production of the type II emission. We obtain similar results from a comparison between the shock model and the radio observations from NRH. In this case we find that the positions of the radio sources are projected near strong shock regions with oblique to quasi-perpendicular shock geometry.

Typically when shock waves interact with streamers where the Alfvén speed is low, the plasma density is high, and the magnetic field is weak, their strength can be strongly enhanced. As a consequence, if a fast-mode wave is not a shock, its propagation inside the streamer could favor the shock formation, or if the wave is a subcritical shock, it can lead to the formation of a supercritical shock. In any case, this scenario increases the shock strength and consequently can favor electron beam acceleration and excitation of Langmuir waves 
necessary for the production of the type II emission. It is also worth noting that the closed field topology inside the streamers could also have an additional important effect on the efficiency of particle acceleration. For example, at closed magnetic field lines where both loop legs are connected to the shock surface, the natural trapping of particles, a so-called collapsing trap geometry (e.g., Magdalenić et al. 2002), and the continuous reacceleration of particles from the shock could lead to a significant energization.

The connection of the shock model to the evolution of the type II emission from the synthetic spectra not only provides important information about the shock/coronal conditions necessary for the production of type II radio emission but also reproduces well the main features observed in the radio spectra (e.g., the LFML and HFMR in Figure 7), with the limitations explained earlier. However, there are a few points that need some further clarification. A first aspect is that the synthetic spectra failed to capture in fine detail the complex type II emission at the start of the event. At this stage (from 09:43 to 09:48 UT) multiple lanes of the radio emission are observed. These emission lanes do not constitute classical pairs of bandsplit emission, as they are morphologically very different. For those lanes it is difficult to conclude at which parts of the shock they are generated. For the type II emission after 09:48 UT the two observed lanes most probably constitute a pair of classical band split. We find a lot of similarities between morphology of the emission of high- and low-frequency lanes (see Figure 2). From the frequency ratio of the two bands we find a density compression ratio of around 1.54 (ranging from 1.4 to 1.8). Similar values have been reported by previous statistical analysis (e.g., Vršnak et al. 2001) for type II bursts at the same height. From the shock model we find that, at the same time interval, the compression ratio varies globally between a range of 1.4 and 2.9, with a medial value of around 2.2. We find that the modeled compression ratio is on average higher than the values derived from the observations of the type II band split for this event. It is not completely clear whether this difference is induced by the model or is related to observations. We note that previous studies have shown a general consistency between the $M_{\mathrm{A}}$ and $X$ values derived from the type II band split and other observational methods (e.g., Frassati et al. 2019; Maguire et al. 2020).

The synthetic spectra show an additional lane in high frequencies (HFML in Figure 7) that it is not observed in the radio spectrum. There are a few possible scenarios that could explain this discrepancy. A main observation that comes from the modeling is that the shock regions responsible for the production of this feature in the synthetic spectra are mostly subcritical and do not produce strong enough beams necessary for production of radio emission. Another possibility includes a failure of the MHD model to capture realistically the coronal conditions at some regions during the shock expansion, however, there is no self-consistent way to tell whether the MHD model does not reproduce realistically the coronal conditions for some regions. Another aspect is that the methods used to produce the synthetic spectra are too simplistic to capture in detail the production of the radio emission. For example, for the harmonic emission perhaps a lacking ingredient is the necessity of counterstreaming electrons to produce counterpropagating Langmuir waves that have not been accounted for (e.g., Ganse et al. 2014).
With the results discussed above, we have improved our understanding of the shock wave and the ambient coronal conditions for the occurrence of type II radio bursts in the low corona. In a follow-up study we will examine the shock conditions for type II radio emission at longer hectometric wavelengths.

\section{Conclusions}

In this study we modeled a shock wave associated with a solar event and a complex type II radio burst. We performed a detailed comparison between the shock model and the radio observations in the metric and decametric range. Our key findings can be summarized as follows:

1. We show that the shock formation precedes the start of the type II radio burst; however, the shock wave is then subcritical and electron acceleration is at that time too weak to produce radio emission.

2. We show that the start of the type II radio burst occurs about when the shock becomes supercritical. The early intense phase of the type II burst occurs when the shock is mostly confined to closed field regions. This likely explains why no escaping energetic electrons are observed as additional type III emissions. Part of the shock wave interacts with a streamer, and the shock geometry is mostly quasi-perpendicular at this time.

3. We show that type II radio emission most probably originates, throughout the event, from regions where the shock wave is strong and supercritical, with relatively high density compression ratio and quasi-perpendicular geometry (high $\theta_{\mathrm{BN}}$ ). This strongly supports the notion that shock drift acceleration generates the electron beams that are ultimately responsible for type II radio emission. A comparison between the shock model and the radio observations from NRH confirms that the radio sources are situated near strong shock regions with oblique to quasi-perpendicular shock geometry.

4. The evolution of the shock wave inside a streamer seems to play a very important role in the enhancement of the shock strength and the formation of a supercritical shock. Most importantly, we find that this condition will favor the efficient electron beam acceleration by the shock and the production of type II radio emission.

The IRAP team acknowledges support from the French space agency (Centre National des Etudes Spatiales; CNES; https://cnes.fr/fr) that funds activity in plasma physics data center (Centre de Données de la Physique des Plasmas; CDPP; http://cdpp.eu/) and the Multi Experiment Data \& Operation Center (MEDOC), the Solar-Terrestrial Observations and Modelling Service; (STORMS;https://storms-service.irap. omp.eu). This includes funding for the data mining tools AMDA (http://amda.cdpp.eu/), CLWEB (clweb.cesr.fr/), and the propagation tool (http://propagationtool.cdpp.eu). A.K. acknowledges financial support from the ANR COROSHOCK project (ANR-17-CE31-0006-01). The work of A.W. was supported by DLR under grant No. 50 QL 1701. I.C.J. was supported by a PhD grant awarded by the Royal Observatory of Belgium. J.M. acknowledges funding by the BRAIN-be (Belgian Research Action through Interdisciplinary Networks) project CCSOM (Constraining CMEs and Shocks by Observations and Modelling throughout the inner heliosphere) and the 
BRAIN-be project SWiM (Solar Wind Modeling with EUHFORIA for the new heliospheric missions). R.V. acknowledges the financial support of the Academy of Finland (projects 309939 and 312357). We thank the RSDB service at LESIA/ USN (Observatoire de Paris) for making the NRH/ORFEES/ NDA data available and the radio monitoring service at LESIA (Observatoire de Paris) for providing value-added data that have been used for this study. We are grateful to Nancay Radioheliograph and Nancay Decameter Array operated by the Observatoire de Paris and funded by the French research agency CNRS/INSU and the ORFEES radio spectrograph (Observations Radio pour Fedome et IEtude des Eruptions Solaires), which is the result of a partnership between the Paris Observatory and the Air Force French, for giving access to the data. We thank FHNW, Institute for Data Science in Brugg/Windisch, Switzerland, for hosting the e-Callisto network. We thank the STEREO: SECCHI, S/WAVES; SOHO: LASCO; Wind/WAVES; and SDO/AIA teams and Predictive Science Inc. for providing the data used in this study. The STEREO SECCHI data are produced by a consortium of RAL (UK), NRL (USA), LMSAL (USA), GSFC (USA), MPS (Germany), CSL (Belgium), IOTA (France), and IAS (France). SOHO is a mission of international cooperation between ESA and NASA. The SDO/AIA data are provided by the Joint Science Operations Center (JSOC) Science Data Processing (SDP).

\section{ORCID iDs}

Jasmina Magdalenic (다 https://orcid.org/0000-00031169-3722

Rami Vainio (ib https://orcid.org/0000-0002-3298-2067

\section{References}

Aschwanden, M. J., \& Boerner, P. 2011, ApJ, 732, 81

Aschwanden, M. J., Boerner, P., Schrijver, C. J., et al. 2013, SoPh, 283, 5 Bougeret, J. L., Goetz, K., Kaiser, M. L., et al. 2008, SSRv, 136, 487 Bougeret, J.-L., Kaiser, M. L., Kellogg, P. J., et al. 1995, SSRv, 71, 231 Brueckner, G. E., Howard, R. A., Koomen, M. J., et al. 1995, SoPh, 162, 357 Cairns, I. H., Knock, S. A., Robinson, P. A., et al. 2003, SSRv, 107, 27

Cho, K.-S., Bong, S.-C., Moon, Y.-J., et al. 2011, A\&A, 530, A16 Claßen, H. T., \& Aurass, H. 2002, A\&A, 384, 1098

Frassati, F., Susino, R., Mancuso, S., et al. 2019, ApJ, 871, 212

Ganse, U., Kilian, P., Spanier, F., et al. 2014, A\&A, 564, A15

Gopalswamy, N., Xie, H., Mäkelä, P., et al. 2010, ApJ, 710, 1111

Gopalswamy, N., Yashiro, S., Xie, H., et al. 2008, ApJ, 674, 560

Grechnev, V. V., Lesovoi, S. V., Kochanov, A. A., et al. 2018, JASTP, 174, 46

Grechnev, V. V., Uralov, A. M., Kochanov, A. A., et al. 2016, SoPh, 291, 1173
Holman, G. D., \& Pesses, M. E. 1983, ApJ, 267, 837

Howard, R. A., Moses, J. D., Vourlidas, A., et al. 2008, SSRv, 136, 67

Jebaraj, I. C., Magdalenić, J., Podladchikova, T., et al. 2020, A\&A, 639, A56

Kerdraon, A., \& Delouis, J.-M. 1997, in Coronal Physics from Radio and Space Observations, Lecture Notes in Physics, Vol. 483, ed. G. Trottet (Berlin: Springer), 192

Knock, S. A., Cairns, I. H., Robinson, P. A., et al. 2001, JGR, 106, 25041

Kong, X., Chen, Y., Guo, F., et al. 2015, ApJ, 798, 81

Kouloumvakos, A., Patsourakos, S., Hillaris, A., et al. 2014, SoPh, 289, 2123

Kouloumvakos, A., Rouillard, A. P., Wu, Y., et al. 2019, ApJ, 876, 80

Krupar, V., Magdalenić, J., Eastwood, J. P., et al. 2019, ApJ, 882, 92

Kumar, P., Innes, D. E., \& Cho, K.-S. 2016, ApJ, 828, 28

Leblanc, Y., Dulk, G. A., \& Bougeret, J.-L. 1998, SoPh, 183, 165

Lecacheux, A. 2000, GMS, 119, 321

Lemen, J. R., Title, A. M., Akin, D. J., et al. 2012, SoPh, 275, 17

Lionello, R., Linker, J. A., \& Mikić, Z. 2009, ApJ, 690, 902

Lv, M. S., Chen, Y., Li, C. Y., et al. 2017, SoPh, 292, 194

Magdalenić, J., Marqué, C., Krupar, V., et al. 2014, ApJ, 791, 115

Magdalenić, J., Marqué, C., Zhukov, A. N., et al. 2010, ApJ, 718, 266

Magdalenić, J., Marqué, C., Zhukov, A. N., et al. 2012, ApJ, 746, 152

Magdalenić, J., Vršnak, B., \& Aurass, H. 2002, in Solar Variability: From Core to Outer Frontiers, ed. A. Wilson (Noordwijk: ESA), 335

Maguire, C. A., Carley, E. P., McCauley, J., et al. 2020, A\&A, 633, A56

Mancuso, S., \& Raymond, J. C. 2004, A\&A, 413, 363

Mann, G., \& Classen, T. 1995, AdSpR, 15, 365

Mann, G., Jansen, F., MacDowall, R. J., et al. 1999, A\&A, 348, 614

Mann, G., Melnik, V. N., Rucker, H. O., et al. 2018, A\&A, 609, A41

McLean, D. J. 1967, PASAu, 1, 47

Nelson, G. J., \& Melrose, D. B. 1985, in Solar Radiophysics: Studies of Emission from the Sun at Metre Wavelengths, ed. D. J. McLean \& N. R. Labrum (Cambridge: Cambridge Univ. Press), 333

Nindos, A., Alissandrakis, C. E., Hillaris, A., et al. 2011, A\&A, 531, A31

Nindos, A., Aurass, H., Klein, K.-L., et al. 2008, SoPh, 253, 3

Prakash, O., Umapathy, S., Shanmugaraju, A., et al. 2009, SoPh, 258, 105

Riley, P., Lionello, R., Linker, J. A., et al. 2011, SoPh, 274, 361

Rouillard, A. P., Plotnikov, I., Pinto, R. F., et al. 2016, ApJ, 833, 45

Rušin, V., Druckmüller, M., Aniol, P., et al. 2010, A\&A, 513, A45

Schmidt, J. M., \& Cairns, I. H. 2012, JGRA, 117, A04106

Smerd, S. F., Sheridan, K. V., \& Stewart, R. T. 1974, in IAU Symp. 57, Coronal Disturbances, ed. G. A. Newkirk (Dordrecht: Reidel), 389

Smerd, S. F., Sheridan, K. V., \& Stewart, R. T. 1975, ApL, 16, 23

Suresh, K., \& Shanmugaraju, A. 2015, SoPh, 290, 875

Treumann, R. A., \& Labelle, J. 1992, ApJL, 399, L167

Vršnak, B., Aurass, H., Magdalenić, J., et al. 2001, A\&A, 377, 321

Vršnak, B., \& Lulić, S. 2000, SoPh, 196, 157

Warmuth, A. 2015, LRSP, 12, 3

Warmuth, A., Vršnak, B., Magdalenić, J., et al. 2004, A\&A, 418, 1117

Wild, J. P., \& McCready, L. L. 1950, AuSRA, 3, 387

Wild, J. P., Murray, J. D., \& Rowe, W. C. 1954, AuJPh, 7, 439

Wuelser, J.-P., Lemen, J. R., Tarbell, T. D., et al. 2004, Proc. SPIE, 5171, 111

Zimovets, I., Vilmer, N., Chian, A. C.-L., et al. 2012, A\&A, 547, A6

Zucca, P., Carley, E. P., Bloomfield, D. S., et al. 2014, A\&A, 564, A47

Zucca, P., Morosan, D. E., Rouillard, A. P., et al. 2018, A\&A, 615, A89

Zucca, P., Pick, M., Démoulin, P., et al. 2014, ApJ, 795, 68 